\title{
Intrapartum Amniotic Fluid Volume Estimation in Low-risk Pregnancy as a Predictor of Perinatal Outcome
}

\author{
1J Jeanet, ${ }^{2}$ Santosh Joseph Benjamin, ${ }^{3} \mathrm{Jiji}$ Elizabeth Mathews, ${ }^{4}$ Swati Rathore, ${ }^{5}$ Ajit Sebastian, ${ }^{6}$ Elsy Thomas
}

\begin{abstract}
Objectives: The study was undertaken to determine the efficacy of routine estimation of amniotic fluid volume using the amniotic fluid index (AFI) as a means of predicting an adverse perinatal outcome in low-risk pregnancies.
\end{abstract}

Materials and methods: Five hundred and three singleton lowrisk pregnancies with cephalic presentation between 37 and 40 weeks and 6 days of gestation with intact or ruptured membranes had AFI estimations within 36 hours of delivery.

Results: The frequency of nonreassuring fetal heart patterns and perinatal outcomes among patients with oligohydramnios $(<5 \mathrm{~cm})$, borderline oligohydramnios $(5$ to $8 \mathrm{~cm})$ and normal $(>8 \mathrm{~cm}$ ) liquor were compared and not found to be different. Cesarean section for nonreassuring fetal status was also similar in the three groups.

Conclusion: This study suggests that AFI estimation during labor in low-risk pregnancies does not predict adverse perinatal outcomes. Further studies are needed to verify this impression.

Keywords: Amniotic fluid volume, Amniotic fluid index, Oligohydramnios, Fetal distress, Perinatal outcome, Perinatal asphyxia.

How to cite this article: Jeanet $\mathrm{J}$, Benjamin SJ, Mathews JE, Rathore S, Sebastian A, Thomas E. Intrapartum Amniotic Fluid Volume Estimation in Low-risk Pregnancy as a Predictor of Perinatal Outcome. J South Asian Feder Obst Gynae 2014;6(3):156-158.

\section{Source of support: Nil}

Conflict of interest: None

\section{INTRODUCTION}

It is well recognized that a proportion of patients who are considered not to be at risk during the antenatal period will be at risk during labor and some of their babies may develop asphyxia. ${ }^{1}$ Different tests have been devised to identify patients who may require cesarean delivery in order to avoid fetal asphyxia and assessment

\footnotetext{
1,2,4-6 Assistant Professor, ${ }^{3}$ Professor

${ }^{1-6}$ Department of Obstetrics and Gynecology, Christian Medical College, Vellore, Tamil Nadu, India
}

Corresponding Author: Santosh Joseph Benjamin, Assistant Professor, Department of Obstetrics and Gynecology Christian Medical College, Vellore, Tamil Nadu, India, Phone: 04162286172, e-mail: sbenjamin@cmcvellore.ac.in of amniotic fluid volume (AFV) is one such test. ${ }^{2}$ The rationale for estimating amniotic fluid volume is to diagnose oligohydramnios which has been implicated as an important cause of intrapartum fetal distress and an adverse perinatal outcome. ${ }^{3}$ However, not all reports show that reduction of amniotic fluid is a sufficiently reliable predictor of an unfavorable perinatal outcome. ${ }^{4-9}$

This study was therefore, undertaken to determine the efficacy of routine estimation of AFV as a means of predicting an adverse perinatal outcome in low-risk pregnancies. Among the different methods employed to quantify AFV is the estimation of the amniotic fluid index (AFI) by ultrasonography ${ }^{2}$ and we chose to use this index as a measure of AFV in this study.

\section{MATERIALS AND METHODS}

This prospective observational study was undertaken with the approval of the Institutional Review Board and the Institutional Ethics Committee. Singleton pregnancies with cephalic presentation between 37 and 40 weeks and 6 days of gestation with intact or ruptured membranes were considered for inclusion in the study. Patients with a gestational age exceeding 41 weeks, gestational hypertension, intrauterine growth restriction, preterm premature rupture of membranes, antepartum hemorrhage, malpresentation, history of previous cesarean delivery, fetal congenital anomalies and a prior diagnosis of either oligohydramnios or polyhydramnios were excluded. The patients who fulfilled these criteria were recruited after obtaining informed consent.

Three obstetricians who were not involved in the clinical monitoring of the patients performed ultrasonography using a $3.5 \mathrm{MHz}$ linear transducer on a Logic 100 Pro (GE) machine on all the patients less than 36 hours prior to delivery. AFI was estimated by the four quadrant method of Phelan et al by summating the deepest pocket in each of the quadrants. ${ }^{2}$ An AFI value of $<5 \mathrm{~cm}$ was regarded as oligohydramnios, values between 5.1 and $8 \mathrm{~cm}$ was regarded as borderline oligohydramnios and values between 8 and $20 \mathrm{~cm}$ was regarded as normal. No intervention was carried out on the basis of AFI values and every patient was monitored by continuous external cardiotocogram (CTG) during labor. Labor was induced by artificial rupture of membranes (ARM), 8 to 
12 hours after cervical ripening with prostaglandins. AFI measurements were done in all these patients prior to ARM.

The obstetricians treating the patients and the independent investigator documenting the outcomes $(\mathrm{JJ})$ were unaware of the AFI values. Apgar scoring of the newborn was done 1 and 5 minutes after delivery.

Fetal distress was considered as the primary outcome measure and it was considered to be present if any one or more of the following features were present: recurrent variable deceleration or late deceleration of fetal heart rate, prolonged bradycardia or/and an Apgar score of $<6$ at both 1 and 5 minutes after birth.

The secondary outcome measures were instrumental or cesarean delivery for fetal distress, meconium staining of amniotic fluid, need for amnioinfusion and the need for admitting the newborn in the neonatal intensive care unit (NICU).

\section{Sample Size Calculation}

The required sample size was calculated using the following formula:

$\mathrm{N}=\mathrm{Z}_{1}-\alpha / 2^{2} \mathrm{PQ} / \mathrm{d}^{2}$, where $\mathrm{P}=$ the assumed prevalence $-5 \%$; $\mathrm{Q}=1-\mathrm{P} 95 \%$ and $\mathrm{D}=$ precision or width of $95 \%$ confidence interval (CI) of $2 \%$.

The required sample size based on this calculation was 490 patients and 503 were recruited for the study.

\section{STATISTICAL METHOD}

Statistical analysis was carried using the Statistical Package for Social Sciences (SPSS version 15.0). Categorical variables were compared using the Chi-square test. Analysis of variance (ANOVA) and the t-test were used to compare continuous variables. A p-value of $<0.05$ was considered significant.

\section{RESULTS}

Of the 503 women recruited for the study, 93 (18.49\%) had oligohydramnios, 219 (43.54\%) had borderline oligohydramnios and in the remaining 191 patients $(37.9 \%)$ the AFI values were normal. The age of the patients and the gestational ages were comparable in these three groups with no statistically significant differences. One hundred and seventy-three patients had spontaneous onset of labor while 330 were induced. The frequency of spontaneous labor was 36.6, 33.3 and 34.5\% in the oligohydramnios, borderline and normal groups respectively (these differences were not statistically significant). The frequencies of intact membranes were almost identical for the oligohydramnios and borderline groups (69.9 and 69.4\%), while it was significantly higher in the normal group $(80.6 \%$; p < 0.05). The mean duration of labor and the duration of ruptured membranes were comparable in the three groups. One hundred and thirty-two patients had spontaneously ruptured their membranes prior to AFI estimation; in this group, oligohydramnios was noted in 28, borderline values in 67 and normal values of AFI in 37.

$50.5 \%$ of the patients with AFI $<5 \mathrm{~cm}$ had nonreassuring fetal heart rate recordings while among the borderline and normal AFI groups 53.9 and $46.6 \%$ had abnormal fetal heart recordings. No specific fetal heart abnormality was encountered more frequently in the oligohydramnios or borderline groups (Table 1).

The frequencies of adverse outcomes in the three AFI groups are shown in Table 2; the adverse outcomes were not more frequent among patients with oligohydramnios.

\section{DISCUSSION}

It has been recommended that estimation of AFI should be an integral part of antenatal monitoring of high-risk pregnancies ${ }^{10}$ and a recent study involving a very large number of patients recruited over a 10 years period demonstrated that estimation of AFI is useful in predicting adverse outcomes in preterm pregnancies. ${ }^{11}$ However, the role of estimation of AFI in low-risk pregnancies is less clearly defined.

The results of the present study suggest that estimation of AFI in low-risk pregnancies does not predict adverse outcomes as adverse primary and secondary outcomes were not encountered more frequently in mothers or newborns when the AFI was less than $5 \mathrm{~cm}$. On the contrary, Locatelli et al ${ }^{12}$ suggested that AFI estimation in uncomplicated term pregnancies did have a significant prognostic value. Other studies have also shown an association between oligohydramnios and a poor perinatal outcome. ${ }^{2-7}$

Table 1: Frequency of fetal heart patterns suggestive of fetal distress in the three AFI groups

\begin{tabular}{|c|c|c|c|c|c|c|}
\hline \multirow{2}{*}{\multicolumn{2}{|c|}{ Outcome variable }} & \multirow{2}{*}{\multicolumn{3}{|c|}{ Amniotic fluid index }} & \multirow{2}{*}{$\begin{array}{l}p \text {-value } \\
\leq 5 \mathrm{~cm} \text { vs } \\
8.1-20 \mathrm{~cm}\end{array}$} & \multirow{2}{*}{$\begin{array}{l}p \text {-value } \\
5.1-8 \mathrm{~cm} \text { vs } \\
8.1-20 \mathrm{~cm}\end{array}$} \\
\hline & & & & & & \\
\hline \multirow{4}{*}{$\begin{array}{l}\text { Non- } \\
\text { reassuring } \\
\text { fetal heart } \\
\text { patterns }\end{array}$} & Prolonged bradycardia (38) & $10(10.6 \%)$ & $18(8.2 \%)$ & $10(52 \%)$ & 0.08 & 0.23 \\
\hline & Tachycardia with poor variability (28) & $5(5.3 \%)$ & $7(3.2 \%)$ & $12(6.3 \%)$ & 0.76 & 0.14 \\
\hline & Late deceleration (12) & $1(1.1 \%)$ & $7(3.2 \%)$ & $4(2.1 \%)$ & 0.54 & 0.49 \\
\hline & Variable deceleration $(N=230)$ & $43(46.2 \%)$ & $109(49.8 \%)$ & $78(40.8 \%)$ & 0.39 & 0.07 \\
\hline
\end{tabular}


Table 2: Effect of amniotic fluid volume on different outcome variables

\begin{tabular}{|c|c|c|c|c|c|c|}
\hline \multirow[t]{2}{*}{ Outcome variable } & & \multicolumn{3}{|c|}{ Amniotic fluid index } & \multirow{2}{*}{$\begin{array}{l}p \text {-value } \\
\leq 5 \mathrm{~cm} \text { vs } \\
8.1-20 \mathrm{~cm}\end{array}$} & \multirow{2}{*}{$\begin{array}{l}p \text {-value } \\
5.1-8 \mathrm{~cm} \text { vs } \\
8.1-20 \mathrm{~cm}\end{array}$} \\
\hline & & $\begin{array}{l}<<\mathrm{cm} \\
(n=93)\end{array}$ & $\begin{array}{l}5.1-8 \mathrm{~cm} \\
(n=219)\end{array}$ & $\begin{array}{l}>8 \mathrm{~cm} \\
(n=191)\end{array}$ & & \\
\hline \multirow[t]{2}{*}{ Neonatal outcome } & Apgar score $<6$ & Nil & Nil & Nil & - & \\
\hline & NICU admission & $2(2.1 \%)$ & $2(0.9 \%)$ & $4(2 \%)$ & 0.92 & 0.26 \\
\hline \multirow[t]{2}{*}{ Mode of delivery } & $\begin{array}{l}\text { Cesarean delivery for } \\
\text { fetal distress }\end{array}$ & $12(12.9 \%)$ & $39(17.8 \%)$ & $40(20.9 \%)$ & 0.10 & 0.42 \\
\hline & $\begin{array}{l}\text { Instrumented vaginal delivery for } \\
\text { fetal distress }\end{array}$ & $17(18.3 \%)$ & $35(15.9 \%)$ & $37(19.4 \%)$ & 0.83 & 0.37 \\
\hline \multirow[t]{2}{*}{ Amniotic fluid } & Meconium staining of amniotic fluid & $22(23.6 \%)$ & $44(20.6 \%)$ & $41(21.5 \%)$ & 0.67 & 0.82 \\
\hline & Need for amnioinfusion & $16(17.2 \%)$ & $28(12.8 \%)$ & $32(16.8 \%)$ & 0.92 & 0.263 \\
\hline
\end{tabular}

One of the explanations for our results could be related to the fact that we only included mothers with a gestational age less than 41 weeks. Studies that included mothers with gestational ages exceeding 41 weeks did show significant associations between an unfavorable perinatal outcome and an AFI $<5 \mathrm{~cm}$. 6,12-14

Secondly, in the present study, a single estimate of AFI was obtained. This may be misleading as Divon et $\mathrm{al}^{14}$ demonstrated that serial estimations of AFI may fluctuate and an initial value $>5 \mathrm{~cm}$ may drop below $5 \mathrm{~cm}$ later on. These authors noted a significantly higher frequency of adverse outcomes in patients with final AFI values $<5 \mathrm{~cm}$.

Thirdly, a recent study suggested that the fetal position may influence the AFI value. ${ }^{15}$ We did not record the fetal position and correlate this with the AFI value.

Finally, one limitation of the study was that the accuracy and reproducibility of estimation of AFI was not considered in this study. Though previous studies have demonstrated satisfactory reproducibility of measurement of AFI, it is possible that there could have been a degree of variability in estimation and this could have influenced the results of this study.

Further, studies are need to establish more conclusively the role of routine estimation of AFI in low-risk pregnancies and investigators of such future studies must take into account the sources of potential errors while designing them.

\section{REFERENCES}

1. Heinonon S, Saarikoshi S. Reproductive risk factors for fetal asphyxia at delivery. J Clin Epidemiol 2001;54(4):407-410.

2. Phelan JP, Smith CV, Broussard P, Small M. Amniotic fluid volume assessment with the four quadrant technique at 36-42 weeks' gestation. J Reprod Med 1987;32(7):540-542.

3. Golan A, Lin G, Evron S, Arielli S, Niv D, David MP. Oligohydramnios: maternal complications and fetal outcome in 145 cases. Gynaecol Obstet Invest 1994;37(2):91-95.
4. Rutherford SE, Phelan JP, Smith CV, Jacobs N. The fourquadrant assessment of amniotic fluid volume: an adjunct to antepartum fetal heart rate testing. Obstet Gynecol 1987;70 (3 pt 1):353-356.

5. Sarno AP, Ahn MO, Brar HS, Phelan JP, Platt LD. Intrapartum amniotic fluid volume at term. Association of ruptured membranes, oligohydramnios and increased fetal risk. J Reprod Med 1990 Jul;35(7):719-723.

6. Sarno AP, Ahn MO, Brar HS, Phelan JP, Platt LD. Intrapartum Doppler velocimetry, amniotic fluid volume and fetal heart rate as predictors of subsequent fetal distress. Am J Obstet Gynecol 1989;161:1508-1514.

7. Baron C, Morgan MA, Garite TJ. The impact of amniotic fluid volume assessed intrapartum on perinatal outcome. Am J Obstet Gynecol 1995;173:167-174.

8. Chauhan SP, Cowan BD, Magann EF, Roberts WE, Morrison JC, Martin JN Jr. Intrapartum amniotic fluid index: a poor diagnostic test for adverse perinatal outcome. J Reprod Med 1996;41:860-866.

9. Rainford M, Adair R, Scialli AR, Ghidini A, Spong CY. Amniotic fluid index in uncomplicated term pregnancy. Prediction of outcome. J Reprod Med 2001;46:589-592.

10. Anandakumar C, Biswas A, Arulkumaran S, Wong YC, Malarvishy G, Ratnam SS. Should assessment of amniotic fluid volume form an integral part of antenatal fetal surveillance of high risk pregnancy? Aust NZJ Obstet Gynaecol 1993;33(3):272-275.

11. Petrozella LN, Dashe JS, McIntire DD, Leveno KJ. Clinical significance of borderline amniotic fluid index and oligohydramnios in preterm pregnancy. Obstet Gynecol 2011;117 (2 Pt 1):338-342.

12. Locatelli A, Zagarella A, Toso L, Assi F, Ghidina A, Biffi A. Serial assessment of amniotic fluid index in uncomplicated term pregnancies: prognostic value of amniotic fluid reduction. J Matern Fetal Neonatal Med 2004;15:233-236.

13. Ghosh G, Marsel K, Gudmundsson S. Amniotic fluid index in low-risk pregnancy as an admission test to the labor ward. Acta Obstet Gynecol Scand 2002;81:852-855.

14. Divon MY, Marks AD, Henderson CE. Longitudinal measurement of amniotic fluid index in post-term pregnancies and its association with fetal outcome. Am J Obstet Gynecol 1995;172:142-146.

15. Fok WY, Chan LY, Lau TK. The influence of fetal position on amniotic fluid index and single deepest pocket. Ultrasound Obstet Gynecol 2006;28(2):162-165. 